\title{
A new tool to ensure the fluorescent dye labeling stability of nanocarriers: A real challenge for fluorescence imaging
}

Guillaume Bastiat ${ }^{\mathrm{a}, \mathrm{b}, *}$, Christian Oliver Pritz ${ }^{\mathrm{c}}$, Clemens Roider ${ }^{\mathrm{d}}$, Florian Fouchet ${ }^{\mathrm{a}, \mathrm{b}}$, Erwann Lignières ${ }^{\mathrm{a}, \mathrm{b}}$, Alexander Jesacher ${ }^{\mathrm{d}}$, Rudolf Glueckert ${ }^{\mathrm{c}, \mathrm{e}}$, Monika Ritsch-Marte ${ }^{\mathrm{d}}$, Anneliese Schrott-Fischer ${ }^{\mathrm{c}}$, Patrick Saulnier $^{\mathrm{a}, \mathrm{b}}$, Jean-Pierre Benoit ${ }^{\mathrm{a}, \mathrm{b}}$

a INSERM U 1066, Micro et Nanomédecines biomimétiques-MINT, Angers F-49933, France

b LUNAM Université, UMR-S1066, Angers F-49933, France

' Department of Otolaryngology, Medical University of Innsbruck, Anichstraße 35, 6020 Innsbruck, Austria

d Division of Biomedical Physics, Innsbruck Medical University, Müllerstraße 44, 6020 Innsbruck, Austria

e University Clinics of Innsbruck, Tiroler Landeskrankenanstalten GmbH-TILAK, A-6020 Innsbruck, Austria

\section{A R T I C L E I N F O}

\section{Article history:}

Received 10 December 2012

Accepted 10 June 2013

Available online 19 June 2013

\section{Keywords:}

Lipid nanocapsule

Fluorescent dye

Labeling of nanocarrier

Light scattering

Cell culture

\begin{abstract}
A B S T R A C T
Numerous studies on nanocarriers use fluorescent dye labeling to investigate their biodistribution or cellular trafficking. However, when the fluorescence dye is not grafted to the nanocarrier, the question of the stability of the labeling arises. How can it be validated that the fluorescence observed during an experiment corresponds to the nanocarriers, and not to the free dye released from the nanocarriers? Studying the integrity of the labeling is challenging. Therefore, an innovative approach to confirm the labeling stability was developed, based on the transfer of a fluorescent dye from its hosting nanocarrier to a lipophilic compartment. Lipid nanocapsules (LNC) and triglyceride oil were used as models. The protocol involved mixing of LNC suspension and oil, and then separation by centrifugation. The quality of the separation was controlled by light scattering, using the derived count rate tool. Dye transfer from loaded LNCs to the lipophilic compartment or from a lipophilic compartment containing dye to non-loaded LNC was investigated by varying the nature of the dye and the oil, the oil volume and the LNC dilution. Tensiometry was used to define the dye location in the nanocarrier. Results showed that when dyes such as Nile Red and Coumarin-6 are located in oily core, the transfer occurred in a partition-dependent manner. In contrast, when the dye was entrapped in the surfactant shell of LNCs such as lipophilic indocarbocyanines (i.e. DiO, Dil and DiD), no transfer was observed. Dye diffusion was also observed in cell culture, with Nile Red inside lipid bodies of HEI-OC1 cells, without uptake of LNCs. In contrast, DiO-loaded LNCs had to be internalized to observe fluorescence inside the cells, providing a further confirmation of the absence of transfer in this case, and the stability of fluorescence labeling of the LNCs.
\end{abstract}

(c) 2013 Elsevier B.V. All rights reserved.

\section{Introduction}

The use of fluorescent dyes in the pharmaceutical field is increasingly common due to the development of novel in vivo imaging techniques $[1-3]$. In the last few years, the indocarbocyanine dye family emerged with interesting properties for in vivo imaging, with wavelengths for excitation and emission in the near-infrared window [4]. These recent techniques complete the in vitro tools such as fluorescence and confocal microscopy. The scientific publications dealing with the topic of in vivo fluorescence imaging increased by 7-fold in the last 10 years, with about 1100 publications in 2011 (webofknowledge.com source). Nevertheless, a major issue with the use of fluorescent dyes

\footnotetext{
* Corresponding author at: MINT-UMR_S1066, Institut de Biologie en Santé-IRIS, 4 rue Larrey CHU, 49933 Angers Cedex 9, France. Tel.: +332 44688531; fax: + 332 44688546.

E-mail address: guillaume.bastiat@univ-angers.fr (G. Bastiat).
}

for nanocarrier visualization, in both in vitro and in vivo studies, is determining if the observed fluorescence does in fact correspond to the nanocarrier, not the dye released from the nanocarrier. For example, Almouazen et al. observed the in vivo capture of Nile Red (NR)-loaded nanoparticles into infiltrated macrophages after intratumoral injection, confirmed by the presence of NR inside the macrophages [5]. Gaumet et al. suggested the accumulation of poly (D,L-lactide-co-glycolide) (PLGA) nanoparticles inside Caco-2 cells and their nuclei with the visualization of 3,3'-Dioctadecyloxacarbocyanine perchlorate (DiO) using confocal microscopy, after incubation of DiO-loaded PLGA nanoparticles with Caco-2 cells [6]. In vitro and in vivo stabilities of labeling, i.e. the stability of the dye encapsulation, have to be assessed to locate nanoparticles in biological samples without doubt.

To overcome this problem, some authors used fluorescent dyes covalently grafted to nanocarriers, assuming the absence of cleavage in vivo. Jatariu Cadinoiu et al. used a fluorescein-marked polymer to study the nanoparticle biodistribution after intraperitoneal and intravenous 
administration [7]. Ding et al. grafted NIR-797-isothiocyanate to hydrophilic nanoparticles to study the penetration and accumulation in tumors with real-time near infrared fluorescence imaging [8]. A drawback of the covalent binding of the dye can be the change in physicochemical properties such as solubility or hydrophobic-hydrophilic balance of the components, changing the assembly which stabilizes the nanocarrier. Another method used was the repetition of studies with different probes. Paillard et al. studied lipid nanocapsule (LNC) endo-lysosomal escape in malignant rat-glioma cells (F98) [9]. Various probes were used: NR and technetium for fluorescence- and radiolabeling, respectively, showing complementary results regarding LNC cellular uptake, LNC subcellular distribution and lysosomal escape. The drawback of this approach is the time required to multiply experiments.

Assessing the stability of the labeling of a nanocarrier must be prioritized in characterization purposes. This task becomes challenging when a lipophilic dye is solubilised inside a lipophilic nanocarrier, as in the case with LNC. Classical separation like dialysis against water or aqueous buffer is difficult to perform. From a thermodynamic point of view, the lipophilic dye would remain inside the lipophilic nanocarrier and would not be transferred to the external aqueous medium. In addition, the disruption of the nanocarrier could be the cause of a loss of labeling stability with dye release and aggregation in water.

This current study reports on the development of a new approach to investigate the labeling stability when a lipophilic fluorescent dye is encapsulated inside a lipophilic nanocarrier. The LNCs are an appropriate model for lipophilic nanocarriers. LNCs are a well established system and were first patented by Heurtault et al. [10]. These nanocarriers, with a hydrodynamic diameter ranging from 20 to $120 \mathrm{~nm}$, can mimic lipoproteins. LNCs are generally composed of an oily core (medium-chain triglycerides) surrounded by a mixed layer of lecithins and a pegylated surfactant [11]. Due to their PEGylated shell and their versatility in terms of surface modifications, LNCs can provide an interesting platform for nanomedicine [12]. The oily core of LNCs allows the easy incorporation of lipophilic dyes for labeling. The experimental protocol involved mixing of a LNC suspension and a lipophilic external compartment (LC) (oil for example), followed by a separation using slow centrifugation. The quality of separation was assessed by quantifying the LNC concentration (in LNC suspension) with an innovative method. Various hydrophobic fluorescent dyes were tested and some of the mixture parameters were changed: LNC size, LNC suspension/LC ratio, nature of LC, dye concentration, etc. The dye transfer studies were performed with two different methods: directly from dye-loaded LNC to LC, and reverse method from dye solubilised in LC to non-loaded LNC. The results gave indications about dye localization inside the LNCs, which were confirmed by interfacial tension measurements. Finally, the approach was tested in cell culture, and confirmed the hypotheses made on the various labeling stabilities of LNCs.

\section{Materials and methods}

\subsection{Materials}

Lipoïd ${ }^{\circledR}$ S75-3 (soybean lecithin at $69 \%$ of phosphatidylcholine and $10 \%$ of phosphatidylethanolamine) (Lip) and Kolliphor ${ }^{\circledR}$ HS15 (formerly Solutol® HS15; mixture of free polyethylene glycol 660 and polyethylene glycol 660 hydroxystearate) (Kol) were kindly supplied by Lipoïd GmbH (Ludwigshafen, Germany) and BASF (Ludwigshafen, Germany), respectively. Labrafac® WL 1349 (caprylic-capric acid triglycerides) (Labrafac) was generously provided by Gattefossé S.A. (Saint-Priest, France). $\mathrm{NaCl}$ was purchased from Prolabo (Fontenay-sous-bois, France). Deionized water was obtained from a Milli-Q plus ${ }^{\circledR}$ system (Millipore, Bilerica, USA). Captex® 200 (propylene glycol dicaprylocaprate) (Captex200), Captex ${ }^{\circledR} 300$ (glyceryl tricaprylate/tricaprate) (Captex300) were provided by Abitech Corp. (Columbus, OH, USA) and perfluoro15-crown-5-ether (PFCE) by Chems GmbH (Regenstauf, Germany). Coumarin-6 (6-Cou), Nile Red (NR) and ethyl oleate (EO) were purchased from Sigma-Aldrich (St. Quentin Fallavier, France). 1,1'-dioctadecyl-3,3,3',3'-tetramethylindocarbocyanine perchlorate (DiI), 1,1'-dioctadecyl-3,3,3',3'-tetramethylindodicarbocyanine 4-chlorobenzenesulfonate (DiD) and 3,3'-dioctadecyloxacarbocyanine perchlorate ( $\mathrm{DiO})$ (lipophilic carbocyanine dye family) were provided by Molecular probes ${ }^{\circledR}$ (Eugene, OR, USA).

\subsection{Lipid nanocapsule formulation}

LNC formulation was based on a phase inversion process and has been thoroughly described [10,11]. The quantities of Labrafac (oil phase), water, $\mathrm{NaCl}$ (aqueous phase), Kol and Lip (surfactants) for each formulation were precisely weighted. For $27 \mathrm{~nm}$ LNC, $\mathrm{m}_{\text {Labrafac }}=0.846, \quad \mathrm{~m}_{\text {Kol }}=1.934, \quad \mathrm{~m}_{\text {Lip }}=0.075, \quad \mathrm{~m}_{\text {water }}=2.055$ and $\mathrm{m}_{\mathrm{NaCl}}=0.103 \mathrm{~g}$; for $65 \mathrm{~nm} \mathrm{LNC}, \mathrm{m}_{\text {Labrafac }}=1.028, \mathrm{~m}_{\mathrm{Kol}}=0.846$, $\mathrm{m}_{\text {Lip }}=0.075, \mathrm{~m}_{\text {water }}=2.962$ and $\mathrm{m}_{\mathrm{NaCl}}=0.148 \mathrm{~g}$; for $110 \mathrm{~nm} \mathrm{LNC}$, $\mathrm{m}_{\text {Labrafac }}=1.209, \mathrm{~m}_{\mathrm{Kol}}=0.484, \mathrm{~m}_{\text {Lip }}=0.075, \mathrm{~m}_{\text {water }}=3.143$ and $\mathrm{m}_{\mathrm{NaCl}}=0.157 \mathrm{~g}$.

For dye-loaded LNCs, a fluorescent dye (NR, 6-Cou, DiI, DiO and DiD) was added at concentration of $1,0.5$ and $0.1 \mathrm{mg} \cdot \mathrm{g}^{-1}$ (weight ratio dye/Labrafac). Mixtures were heated to $95{ }^{\circ} \mathrm{C}$ at a rate of $5{ }^{\circ} \mathrm{C} \cdot \mathrm{min}^{-1}$ under magnetic stirring followed by cooling at the same rate to $50{ }^{\circ} \mathrm{C}$. This cycle was repeated three times and the LNC suspension returned at room temperature at the end of the last cycle. LNC suspensions were dialyzed ( $15 \mathrm{kDa}$ cut-off) and filtered $(0.2 \mu \mathrm{m})$ before use. LNC suspension was diluted by factor $60(\mathrm{v} / \mathrm{v})$ before hydrodynamic diameter (Z-Ave), polydispersity index (PdI) and derived count rate (DCR) measurements, to confirm the LNC presence in suspensions. Data are reported in supplementary information table S1.

\subsection{Separation process}

Aqueous LNC suspensions were mixed with oil, acting as the lipophilic compartment (LC). The mixtures were vortexed during $15 \mathrm{~s}$ at room temperature, then centrifuged (4000 rpm, $30 \mathrm{~min}$ ). Once complete separation was obtained, Z-Ave, PdI and DCR were determined for the LNC suspension phase. Fluorescence intensities were measured on LNC suspension and LC phases. Each experiment was repeated 3 times.

Various parameters were modified: (i) nature of the fluorescent dye: NR, 6-Cou, DiI, DiO and DiD; (ii) dye concentration: 1, 0.5 and $0.1 \mathrm{mg} \cdot \mathrm{g}^{-1}$ (weight ratio dye/Labrafac in LNC or Labrafac as LC); (iii) nature of oil as LC: Labrafac, Captex200, Captex300, EO and PFCE; (iv) aqueous LNC suspension dilution: no dilution, 2 and 3.5 dilution factors $(v / v)$; (v) LNC Z-ave: 27, 65 and $110 \mathrm{~nm}$; and (vi) weight ratio $\mathrm{R}$ corresponding to the ratio of Labrafac in $\mathrm{LNC} / \mathrm{Labrafac}$ as LC.

In addition, the dye transfer process was performed according to two protocols. The direct method corresponded to the dye transfer from LNC to LC, with dye-loaded LNC aqueous suspension and non-loaded LC. The reverse method corresponded to the dye transfer from LC to LNC, with non-loaded LNC aqueous suspension and dye-loaded LC (dye solubilized in oil as LC). Transfer protocols and separation process are illustrated in Fig. 1.

\subsection{Hydrodynamic diameter, PdI and Derived Count Rate (DCR) measurements}

The hydrodynamic diameter Z-ave, PdI and DCR of LNCs were determined by quasi-elastic light scattering using a Zetasizer® Nano Series DTS 1060 (Malvern Instrument Ltd, Worcestershire, UK). The Helium-Neon laser, $4 \mathrm{~mW}$, operates at $633 \mathrm{~nm}$, with the scatter 

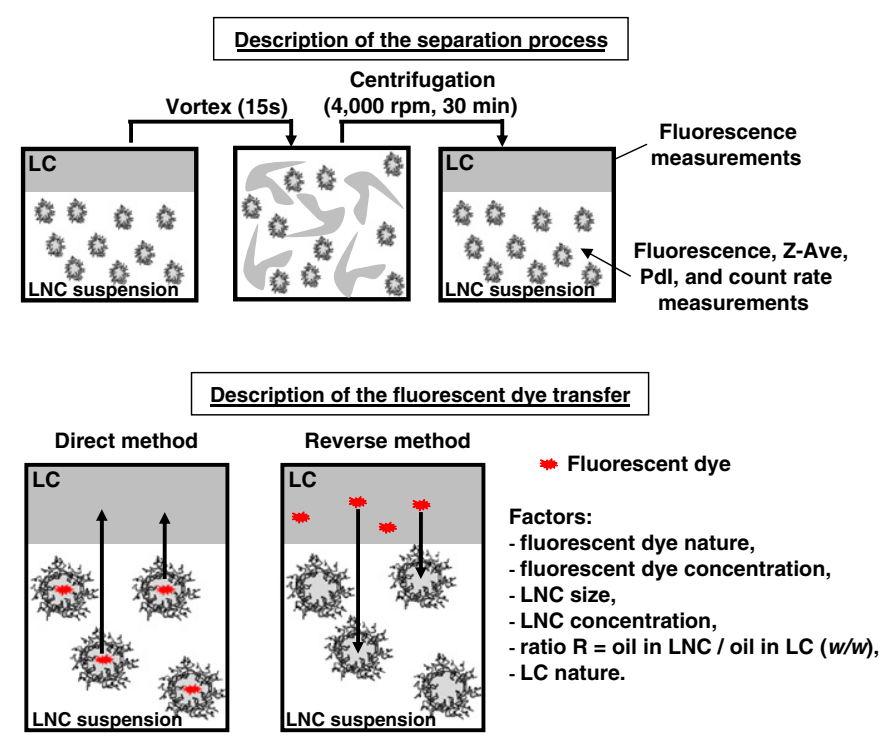

Fig. 1. Separation process and fluorescent dye transfer descriptions.

angle fixed at $173^{\circ}$, at a temperature of $25^{\circ} \mathrm{C}$. The curve fittings of the correlation functions were performed using an exponential fit (Cumulant approach) for Z-ave and PdI determinations for LNC suspensions. DCR can be calculated from the measured count rates of the scattered light on the detector and the attenuation factor (linked to the attenuator value) according to the relation:

$\mathrm{DCR}=\frac{\text { Measured count rate }}{\text { Attenuation factor }}$.

For attenuator values of 1 to 11 , attenuation factors are $8.66 \times 10^{-6}$, $8.26 \times 10^{-5}, 1.23 \times 10^{-4}, 6.01 \times 10^{-4}, 1.42 \times 10^{-3}, 3.62 \times 10^{-3}$, $1.26 \times 10^{-2}, 0.044,0.111,0.281$ and 1 (given by Malvern Instrument Ltd). LNC suspensions were diluted in pure water and DCRs were measured vs. LNC suspension concentrations.

\subsection{Fluorescence measurements}

Fluorescence measurements were assessed on dye-loaded LC phases and dye-loaded LNC suspensions, before and after the transfer, using a microplate reader Fluoroscan Ascent ${ }^{\circledR}$ (Labsystems SA, CergyPontoise, France). Filter pairs with adequate excitation and emission wavelength couples $\left(\lambda_{\mathrm{ex}}-\lambda_{\mathrm{em}}\right)$ were used. These couples were 515590 for NR, 485-518 for 6-Cou, 544-590 for Dil, 485-518 for DiO and 646-678 nm for DiD. Titration curves with dye-loaded LNCs and dye solubilized in LC were performed before evaluating the concentration of dye in each phase after transfer.

\subsection{Interfacial tension measurements}

Adsorption kinetics were obtained at Labrafac-water interface by means of a rinsing drop method with a drop tensiometer device (Tracker Teclis, Longessaigne, France). For this study, fluorescent dyes (NR, 6-Cou, DiI and DiD) were diluted in Labrafac, from 1 to $3 \mathrm{mg} \cdot \mathrm{g}^{-1}$ (weight ratio dye/Labrafac). Basically, a Labrafac drop $(5 \mu \mathrm{L})$ was formed using an Exmire microsyringe (Prolabo, Paris, France) into an optical glass bowl (Hellma, France) containing the water phase. The axial symmetric shape (Laplacian profile) of the drop was analyzed by use of a video camera connected to a microcomputer. From numerical image analysis, with the Laplace equation integrating the drop profile points, the interfacial tension, the surface area, and the volume of the drop were recorded in real time (five measurements per sec). Movements of the piston of the syringe were controlled by a stepping motor connected to a microcomputer, to control the drop volume and to keep the surface area constant.

Adsorption kinetics consisted to the formation of a monolayer by the gradual diffusion of the amphiphilic molecules from the drop core to the interface, until complete saturation was achieved. Saturation was reached when surface tension was stabilized. For each fluorescent dye and each concentration, experiments were performed in triplicate.

\subsection{Interaction studies of LNCS with HEI-OC1 auditory cell line}

HEI-OC1 cells, which are derived from the organ of corti [13], were grown at $33{ }^{\circ} \mathrm{C}$ and $10 \% \mathrm{CO}_{2}$ in DMEM high glucose (Invitrogen, Lofer, Austria, 21063-029) free of phenol red, including 10\% fetal calf serum (Thermo Scientific, USA, SH30071.03, Lot\# ATJ33161) and $50 \mathrm{U} \cdot \mathrm{mL}^{-1}$ of recombinant murine interferon-gamma (Peprotech, London, UK, 315-05). Cells were plated for light microscopy on poly-D-lysine (Sigma, Vienna, Austria, P0296) coated coverslips and for transmission electron microscopy on type I collagen (Becton Dickinson, Maryland, USA, 354236) coated $\mu$-dishes (Ibidi, Martinsried, Germany, 80131). Cells were plated in triplicate $12 \mathrm{~h}$ before the beginning of the experiments and subsequently incubated with LNCs in final concentration of $10^{14}$ particles $\cdot \mathrm{mL}^{-1}$ for 1 and $8 \mathrm{~h}$ in culture conditions or for $1 \mathrm{~h}$ at $4{ }^{\circ} \mathrm{C}$ to prevent endocytosis. After incubation with LNCs, the cells were washed in PBS and subsequently fixed in $4 \%$ paraformaldehyde in PBS for $1 \mathrm{~h}$ at $4{ }^{\circ} \mathrm{C}$, washed again, DAPI stained and mounted in Vectashield ${ }^{\circledR}$ (Vector laboratories, Peterborough, United Kingdom) for fluorescence microscopy. For Coherent Anti-Stokes Raman Scattering (CARS) microscopy, cells were mounted in PBS.

\subsection{Confocal laser scanning microscopy}

Confocal laser scanning microscopy was performed with a LEICA SP5 (LEICA, Mannheim Germany) using LEICA LAS AF software. Images were recorded using a 63 fold/1.4 NA objective. DAPI was excited with a $405 \mathrm{~nm}$ laser and the resulting fluorescence was collected between 420 and $480 \mathrm{~nm}$. Fluorescence originating from DiO, which was excited by a $488 \mathrm{~nm}$ laser, was recorded from 500 to $690 \mathrm{~nm}$ for fluorescence profiling in overlapping $15 \mathrm{~nm}$ steps and from 500 to $545 \mathrm{~nm}$ for imaging. NR was excited with a $514 \mathrm{~nm}$ laser and resulting NR emission was recorded from 530 to $690 \mathrm{~nm}$ in overlapping $15 \mathrm{~nm}$ steps to obtain emission profiles and from 560 to $630 \mathrm{~nm}$ for imaging.

\subsection{Coherent anti-stokes Raman scattering and transmission electron microscopies}

Coherent Anti-Stokes Raman Scattering (CARS) microscopy directly probes the presence of specific molecular resonances and thus allows discriminating between different substances without using dyes [14]. CARS images were taken with a home-built widefield CARS microscope (see [15] for a detailed description of the set-up) using a 40 fold/0.6 NA objective and an Andor iXon 888 EMCCD camera. Each sample section was imaged twice, with the excitation laser frequencies set on- and off-resonant (2850 and $2800 \mathrm{~cm}^{-1}$ wavenumbers) to the stretching mode of $\mathrm{C}-\mathrm{H}$ bonds. The final image is the difference between both recordings, for the sake of suppressing undesired signal components such as dark noise and non-resonant background. Overlays between the CARS and confocal fluorescence images were obtained with the MATLAB image registration tool. The laser vacuum wavelengths for the Stokes and pump beams were $1064 \mathrm{~nm}$ and $816 \mathrm{~nm}$ for the resonant setting and $1064 \mathrm{~nm}$ and $820 \mathrm{~nm}$ for the non-resonant one.

Sample preparation for transmission electron microscopy (TEM) was performed as described previously [16]. 


\subsection{Statistical analysis}

Normal distribution was assumed for the dye proportion in LNC suspension after transfer. Significant differences between means of dye proportion in LNC were analyzed by a one-way analysis of variance (ANOVA 1F), followed by Tuckey-Kramer's post-hoc test for pairwise comparisons.

\section{Results}

\subsection{Blank and dye-loaded LNCS and separation protocol}

The phase-inversion temperature method was first described by Shinoda and Saito [17]. Heurtault et al. used this concept to obtain nano-objects based on an oily core (Labrafac) surrounded by an organized membrane made up of lecithin molecules (Lip) and PEGylated surfactants (Kol), with PEG chains towards the aqueous phase $[10,11]$. The Labrafac and Kol compositions were changed and after temperature cycles, LNC-hydrodynamic diameters (Z-Ave) were modified: 27, 65 and $110 \mathrm{~nm}$, with a low standard deviation $(<2 \mathrm{~nm})$. PdI was lower than 0.1 for all these nano-objects, characteristic to a monomodal and narrow size distribution.

To obtain fluorescent dye-loaded LNCs, fluorescent dyes (NR, 6-Cou, DiD, Dil and DiO) were added to the initial mixture before temperature cycling. Dye concentrations were ranging from 0.1 to $1 \mathrm{mg} \cdot \mathrm{g}^{-1}$ (weight ratio dye/Labrafac). Nano-objects were obtained with a similar size to the corresponding non-loaded counterparts. PdI was also found lower than 0.1 regardless of the dye nature and concentration used. After LNC suspension purification with dialysis and filtration, no free fluorescent dye aggregate and no fluorescent dye inside Kol micelles were found. Controls were used adding fluorescent dye aggregates and fluorescent dye inside Kol micelles in LNC suspension to confirm the efficacy of the purification. Regardless of the dye concentration, total encapsulation was confirmed for NR, 6-Cou, DiD and Dil-loaded LNC using fluorescence spectroscopy with the dye solubilised in Labrafac (titration curves). The titration curve for DiO solubilised in Labrafac was impossible to set up due to partial recrystallization of DiO in Labrafac at room temperature after solubilization at high temperature. Nevertheless, since no DiO aggregate was observed in DiO-loaded LNC suspension, total encapsulation was assumed and will be explained later.

A new protocol was established to evaluate the transfer of lipophilic fluorescence dye from lipid nanocapsules (LNCs), suspended in aqueous solution, to a lipophilic compartment (LC), i.e. the evaluation of the labeling stability, without any physical barrier to separate the two antagonistic media. Basically, oil (as LC) was added in an aqueous suspension of LNCs. The 2 phases were mixed for $15 \mathrm{~s}$ by vortexing, and then centrifuged ( $4000 \mathrm{rpm}, 30 \mathrm{~min}$ ). A phase separation was obtained where the LC remained at the top and the aqueous suspension of LNCs was located at the bottom. These two phases could be physically separated to perform fluorescence measurements in order to quantify the transfer of fluorescent dye from LNCs to LC.

To confirm that presence of fluorescent dye in LC is due to dye transfer and not to a poor separation between LNC in suspension and LC, LNC concentration before and after centrifugation was determined. The description of the protocol is given in supplementary information (Appendix 1). LNC concentrations in aqueous suspension before and after centrifugation were considered similar, meaning that the totality of LNC was recovered in the aqueous phase and no LNC was present in the oil compartment after separation (Table S1 in Appendix 1). With centrifugation, it was possible to separate the aqueous LNC suspension from the LC after mixture of these 2 media, and to evaluate the fluorescent dye transfer using fluorescence measurements of the two media after physical separation.

\subsection{Nile Red transfer}

Nile Red (NR) transfer, a lipophilic fluorescent dye, was studied in the direct method: from LNCs to Labrafac, used as LC (direct way in Fig. 2). In a second step, the reverse method, NR transfer from Labrafac to blank LNCs was also performed (reversed way in Fig. 2).

First, NR transfer was studied for a given LNC size (Z-Ave = $65 \mathrm{~nm}$ ) without dilution of the LNC aqueous suspension. The dye concentration was modified: $0.1,0.5$ and $1 \mathrm{mg} \cdot \mathrm{g}^{-1}$ (weight concentration $\mathrm{NR} /$ Labrafac in LNC). In addition, the weight ratio R was changed: $1 / 1$, $1 / 2$ and $2 / 1$, corresponding to the weight ratio of Labrafac (in LNC)/ Labrafac (as LC).

With $\mathrm{R}=1 / 1$ and a dye concentration of $1 \mathrm{mg} \cdot \mathrm{g}^{-1}, 31 \pm 2 \%$ of NR was transferred from the LNCs to LC. Furthermore, when NR was loaded in LC at the same concentration, $65 \pm 2 \%$ of the NR was transferred from LC to LNC. The NR distribution in the two compartments appeared to follow a partition coefficient behavior. Whatever the localization of NR before transfer was, about 31-35\% of the total NR was present in Labrafac LC and about $65-69 \%$ of the NR was located in LNC suspension after transfer. As partition coefficients are independent from the concentration of the diffusing compound, the NR concentration should have no effect on the partition values. In order to assess this hypothesis, the NR concentrations were changed to 0.5 and $0.1 \mathrm{mg} \cdot \mathrm{g}^{-1}$. Strikingly no difference in the partition values were observed compared to $1 \mathrm{mg} \cdot \mathrm{g}^{-1} \mathrm{NR}$ concentration, regardless of the initial localization of NR (in LC or LNCs). The partition value for NR was expected to change when the volumes of the media were modified (expressed by the ratio $\mathrm{R}$ between the aqueous suspension and the $\mathrm{LC}$ ). Indeed, when $\mathrm{R}=1 / 2$ (2 more times Labrafac in LC than in LNC), there was a significant difference compared to $\mathrm{R}=1 / 1$ : more $\mathrm{NR}(54 \pm 1 \%)$ was recovered in $\mathrm{LC}$ after transfer. Similar results were obtained for the reverse method, where about $58 \pm 10 \%$ of NR remained in LC. The partition values increased in favor of the increased medium volume. When $\mathrm{R}=2 / 1$ ( 2 times less Labrafac in the LC than in the LNCs), more NR $(88 \pm 5 \%)$ was recovered in the LNCs after transfer.

The effect of the LNC size on the NR transfer was determined with constant weight ratio of Labrafac (in LNC)/Labrafac (as LC) and constant NR concentration: $1 \mathrm{mg} \cdot \mathrm{g}^{-1}$. As depicted in Fig. S2 (Supplementary information), no significant difference was observed when LNC Z-Ave

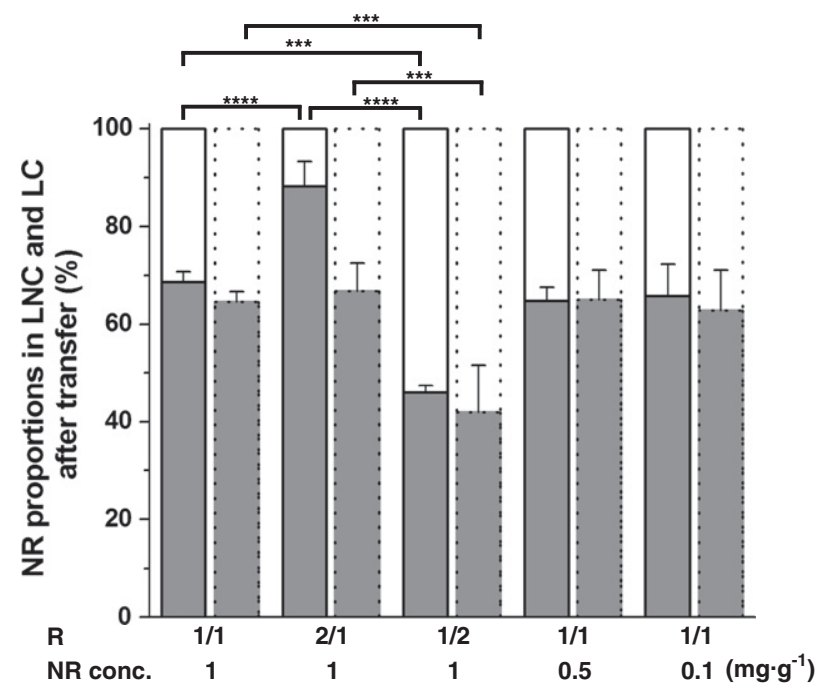

Fig. 2. Proportions of Nile Red (NR) in LNCs (gray part bar) and in LC (white part bar) after the direct method (solid line bar) and the reverse method (dot line bar) transfers (see Fig. 1) vs. NR concentration (NR conc.: dye/Labrafac in LNC $w / w$ ): $1,0.5$ and $0.1 \mathrm{mg} \cdot \mathrm{g}^{-1}$ and $\mathrm{R}$ (Labrafac in LNC/Labrafac as LC $w / w$ ): $1 / 1,1 / 2$ and $2 / 1$. Constant parameters: LNC Z-Ave $=65 \mathrm{~nm}$, LNC concentration $=385 \mathrm{mg} \cdot \mathrm{g}^{-1}$, LC was Labrafac $\left(\mathrm{n}=3\right.$; mean $\left.\pm \mathrm{SD} ;{ }^{* * *}: \mathrm{p}<0.005 ;{ }^{* * *}: \mathrm{p}<0.001\right)$. 
varied from 27 to $110 \mathrm{~nm}$. About 30\% of NR was in Labrafac LC after transfer, regardless of what the initial localization of NR was (in LC or LNC). The partition value was affected by Labrafac volume encapsulated by all LNCs (Fig. 2) but was independent from the Labrafac volume in one individual LNC particle (i.e. individual LNC volume). Finally, for LNC aqueous suspensions, the dilution effect was studied (Supplementary information, Fig. S2) with constant ratio $R=1 / 1$, constant NR concentration of $1 \mathrm{mg} \cdot \mathrm{g}^{-1}$ and constant LNC size (65 nm). When dilutions of aqueous LNC suspensions were applied (dilution factors of 2 and 3.5), less NR (about 23\%) was recovered in Labrafac LC when applied in the direct method, while considering the reverse method, more NR (about 55\%) remained in Labrafac LC, compared to non-diluted LNC aqueous suspension (Fig. 2). These differences were significant.

The transfer process according to the direct method (from LNC to $\mathrm{HC}$ ) was tested changing the nature of the oil as LC (Supplementary information, Fig. S3). Non-diluted LNC (Z-Ave $=65 \mathrm{~nm}$ ) and a constant ratio $R=1 / 1$ was used. NR concentration was fixed at $1 \mathrm{mg} \cdot \mathrm{g}^{-1}$ (dye/Labrafac in LNC). NR proportions in LC after the centrifugation were $25 \pm 2,32 \pm 2,33 \pm 8$ and $3 \pm 5 \%$, for EO, Captex 200 , Captex300 and PFCE as LC, respectively. Similarly to Labrafac, NR transfer was observed from the LNCs to LC for EO, Captex200 and Captex300. Nevertheless, since NR is not soluble in PFCE, this dye remained in totality inside the LNCs (about $97 \pm 5 \%$ ).

\subsection{Coumarin-6, Dil, DiO and DiD transfer}

The transfer process was tested with other hydrophobic fluorescent dyes: 6-Cou, Dil, DiO and DiD. The transfer assessments were performed in both methods (direct and reverse); non-diluted LNCs (Z-Ave $=65 \mathrm{~nm}$ ) were used, LC was Labrafac, the ratio $\mathrm{R}$ was fixed at $1 / 1$ (for all dyes), $1 / 2$ and $2 / 1$ (for 6 -Cou). Dye concentration remained constant at $1 \mathrm{mg} \cdot \mathrm{g}^{-1}$ (dye/Labrafac in LNCs), and was modified for 6-Cou: 0.5 and $0.1 \mathrm{mg} \cdot \mathrm{g}^{-1}$ (6-Cou/Labrafac in LNCs).

For 6 -Cou, with $\mathrm{R}=1 / 1$ and a dye concentration of $1 \mathrm{mg} \cdot \mathrm{g}^{-1}$, $75 \pm 1 \%$ of 6 -Cou remained in LNC (Supplementary information, Fig. S4). Similarly to NR, there was a partitioning for 6-Cou between the LNCs and LC. Nevertheless, the transfer value for the reverse method was different. When 6-Cou was loaded in the LC at the same concentration, $57 \pm 7 \%$ of 6 -Cou was transferred to LNC. 6 -Cou partition was depended on the way of transfer. Similarly to $\mathrm{NR}$, using lower 6-Cou concentration, the proportion of partition remained constant and when $\mathrm{R}$ was modified, proportion of partition was changed (Supplementary information, Fig. S4). Concerning the proportion of 6-Cou in LNC after transfer and separation, $68 \pm 4$ (direct method) and $53 \pm 8 \%$ (reverse method) were found, when 6 -Cou concentration was $0.5 \mathrm{mg} \cdot \mathrm{g}^{-1}$. When 6 -Cou concentration was set to $0.1 \mathrm{mg} \cdot \mathrm{g}^{-1}, 64 \pm 9$ (direct method) and $50 \pm 5 \%$ (reverse method) were observed in LNC. An increase of the LC volume $(\mathrm{R}=1 / 2)$ resulted in a significant lower 6 -Cou proportion in LNC suspension: $47 \pm 13$ after application of the direct method and $40 \pm 10 \%$ for the reverse method, compared to ratio $R=1 / 1$. A decreased LC volume $(R=2 / 1)$ resulted in an equivalent or higher values for 6-Cou proportion in LNC suspension: $70 \pm 3$ for the direct method and $59 \pm 7 \%$ for the reverse method, compared to ratio $\mathrm{R}=1 / 1$.

The opposite results were observed with Dil, DiO and DiD compared to NR and 6-Cou. In the direct method, no transfer was observed from LNC to LC (Fig. 3): $95 \pm 5$ of Dil, $98 \pm 3$ of DiO and $94 \pm 7$ of DiD remained in LNCs after transfer. In the reversed method, no entry of Dil and DiD (about 1\%) in the LNCs was observed. The reverse method experiment was not performed with DiO due to recrystallization in Labrafac at room temperature after high temperature solubilization. Contrary to NR and 6-Cou, DiI, DiO and DiD remained inside LNCs and were not transferred in the presence of a LC around the LNCs. Absence of entry of these dyes inside non-loaded LNCs was also observed.

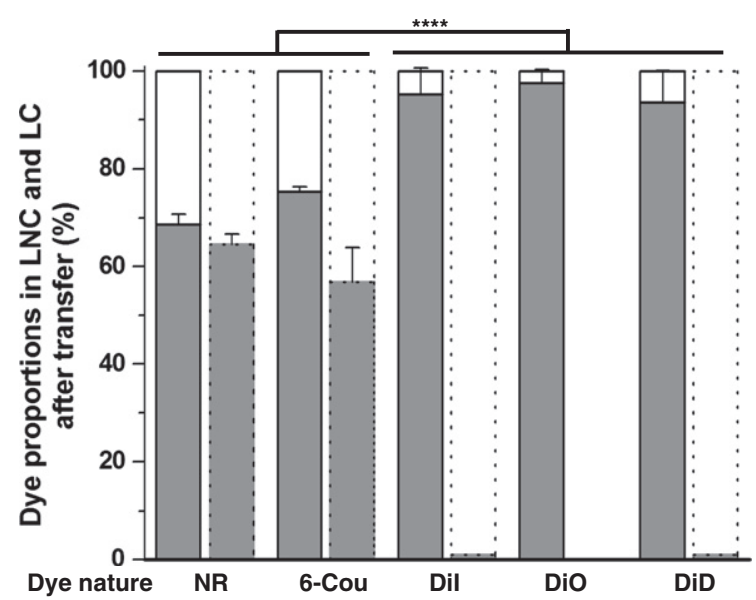

Fig. 3. Proportions of fluorescent dye in LNCs (gray part bar) and in LC (white part bar) after the direct method (solid line bar) and the reverse method (dot line bar) transfers (see Fig. 1) vs. dye nature (NR, 6-Cou, Dil, DiO and DiD). Constant parameters: LNC Z-Ave $=65 \mathrm{~nm}$, LNC concentration $=385 \mathrm{mg} \cdot \mathrm{g}^{-1}$, LC was Labrafac, $\mathrm{R}=1 / 1$ (Labrafac in LNC/Labrafac as LC $w / w$ ) and dye concentration (dye/Labrafac in LNC $w / w)$ was $1 \mathrm{mg} \cdot \mathrm{g}^{-1}$. DiO reversed transfer was not performed $(\mathrm{n}=3$; mean $\pm \mathrm{SD}$; $\left.{ }^{* * *}: \mathrm{p}<0.005 ;{ }^{* * * *}: \mathrm{p}<0.001\right)$.

\subsection{Interfacial tension measurements}

Labrafac-water interface was built with a $5 \mu \mathrm{L}$-Labrafac drop in water. Interfacial tension was measured at the interface vs. dye concentration, when dyes (NR, 6-Cou, Dil and DiD) could be solubilized in Labrafac (Fig. 4). For NR and 6-Cou, a constant interfacial tension was observed, about $30 \mathrm{mN} \cdot \mathrm{m}^{-1}$, independent of the dye concentration in Labrafac (from 0 to $3 \mathrm{mg} \cdot \mathrm{g}^{-1}$ (dye/Labrafac)). These two dyes did not affect the Labrafac-water interface and remained soluble inside the oil drop. For Dil and DiD, when dye concentration increased from 0 to $3 \mathrm{mg} \cdot \mathrm{g}^{-1}$, interfacial tension significantly decreased from 30 to $22 \mathrm{mN} \cdot \mathrm{m}^{-1}$, suggesting that Dil and $\mathrm{DiD}$ dyes behave as amphiphilic molecules and move from the oily core of the drop to the Labrafac-water interface.

\subsection{Single versus double encapsulation}

LNC mixture consisting of a mix of NR-loaded LNCs and DiO-loaded LNCs (Z-Ave $=65 \mathrm{~nm}$; $1 \mathrm{mg} \cdot \mathrm{g}^{-1}$ weight ratio NR or DiO/Labrafac in LNC) was studied. In addition, double encapsulation was also

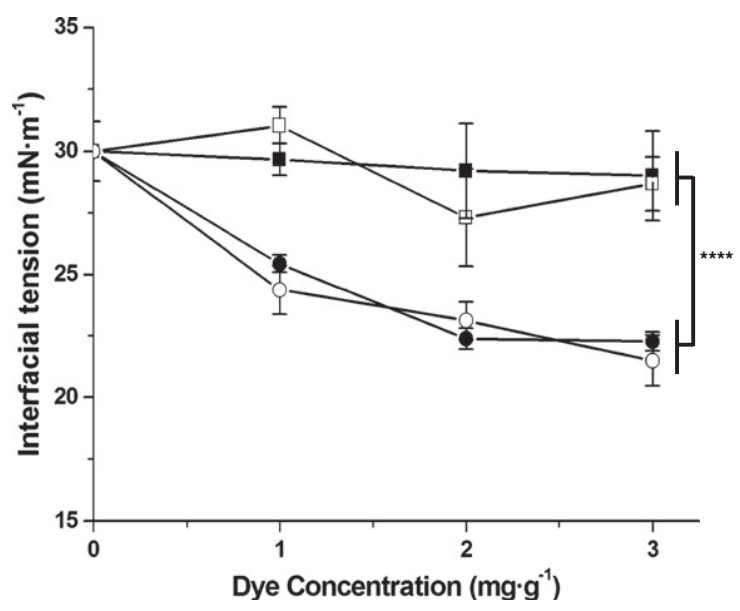

Fig. 4. Interfacial tension of Labrafac-water interface of a $5 \mu \mathrm{L}$-Labrafac drop in water

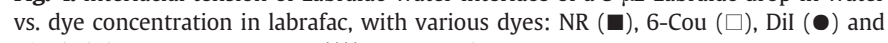
$\operatorname{DiD}(\mathrm{O})\left(\mathrm{n}=3\right.$; mean $\left.\pm \mathrm{SD}{ }^{* * * *}: \mathrm{p}<0.001\right)$. 
performed with NR and DiO in LNCs (Z-Ave $=65 \mathrm{~nm} ; 1 \mathrm{mg} \cdot \mathrm{g}^{-1} \mathrm{NR}$ and DiO/Labrafac in the same LNC). Transfer from LNCs to Labrafac (LC) was performed with a constant ratio $\mathrm{R}=1 / 1$ (Fig. 5). This was studied simultaneously with the 2 dyes: one (NR) which can be transferred from the nanocarrier and the other (DiO) remaining inside. Only this dye couple was studied because there is no overlap of the 2 emission peaks in the fluorescence spectrum. Regardless of the system studied, dyes separately encapsulated and encapsulated together, $29 \pm 1$ and $33 \pm 1 \%$ of transferred NR to LC were observed, respectively. No DiO was recovered in the LC after transfer process for both systems. Results were similar to dye encapsulated alone: DiO-loaded LNCs or NR-loaded LNCs, as in Figs. 2 and 3.

\subsection{Cell culture experiments}

NR and DiO dye separately encapsulated and NR and DiO encapsulated together, as studied previously, were tested in cell culture and incubated with HEI-OC1 cells. Same results were obtained in both systems.

First, HEI-OC1 cells were incubated in DiO/NR-loaded LNCs for $1 \mathrm{~h}$ at $4{ }^{\circ} \mathrm{C}$. Since the low temperature inhibits energy dependent processes, endocytosis of LNCs was prevented. Nonetheless NR staining of dotted structures was observed inside the cells and faint staining of membranes was detected (Fig. 6A-B), while no fluorescence
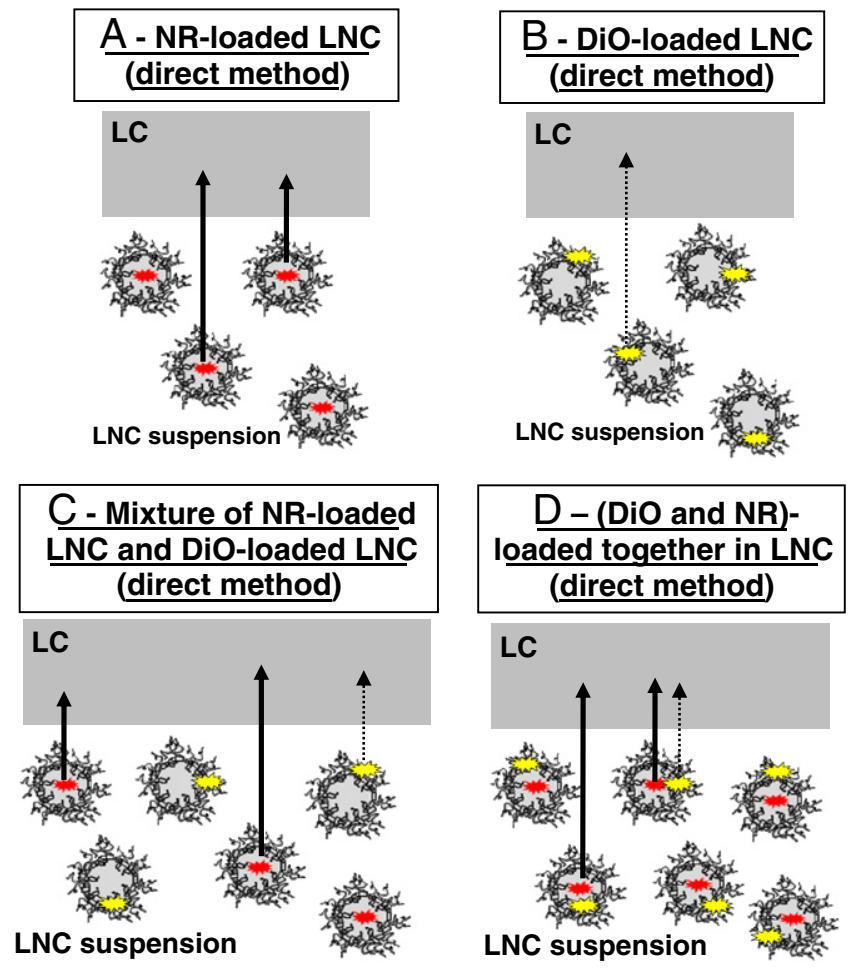

\begin{tabular}{|c|c|c|c|c|}
\hline \multicolumn{3}{|c|}{$\begin{array}{l}\text { Dye proportion in } \mathrm{LNC} \text { after } \\
\text { direct method transfer }(\%)\end{array}$} & \multicolumn{2}{|c|}{$\begin{array}{l}\text { Dye proportion in } \mathrm{LC} \text { after } \\
\text { direct method transfer (\%) }\end{array}$} \\
\hline & NR & $\mathrm{DiO}$ & NR & $\mathrm{DiO}$ \\
\hline A & $69 \pm 2$ & --- & $31 \pm 2$ & --- \\
\hline B & --- & $97.5 \pm 3$ & --- & $2.5 \pm 3$ \\
\hline C & $71 \pm 1$ & $100 \pm 0$ & $29 \pm 1$ & $0 \pm 0$ \\
\hline D & $67 \pm 3$ & $100 \pm 0$ & $33 \pm 3$ & $0 \pm 0$ \\
\hline
\end{tabular}

Fig. 5. NR and DiO proportions after direct method transfer with NR and DiO separately encapsulated: mixture of NR-loaded LNC and DiO-loaded LNC (C), and NR and DiO encapsulated together in the same LNC (D), then compared to dyes encapsulated alone results ( $A$ and $B)$ (as in Fig. 3$)(n=3$; mean $\pm S D)$. originating from DiO was observed inside the cells. NR could diffuse out from the LNCs and stain intracellular lipophilic structures such as lipid bodies and membranes. In order to test this hypothesis, the emission spectra of these putative lipid bodies were analyzed using microscopy. A well-known spectral property of NR is the emission shift towards blue when the dye is dissolved in triglycerides $[18,19]$. Spectral analysis of the sample (excitation $514 \mathrm{~nm}$, spectral scan 530-690 nm) showed bright intracellular dots, suspected to be lipid bodies (Fig. 6A, green arrowheads). They featured exactly that emission shift of the NR emission maximum towards blue (maximum at $570 \mathrm{~nm}$ ), while other membranous structures (Fig. 6A, red arrowhead) were featuring an emission peak at $590 \mathrm{~nm}$ (Fig. 6C).

LNC treated cells were subjected to CARS microscopy in order to assess whether or not these NR positive structures were enriched in aliphatic methyl groups. For that purpose, cells were incubated with NR-loaded LNCs for $1 \mathrm{~h}$ at $4{ }^{\circ} \mathrm{C}$. NR fluorescence was recorded using fluorescence microscopy and subsequently, the same cells were imaged using CARS microscopy. The generated anti-Stokes signal can be considered as a measure for the local density of $\mathrm{C}-\mathrm{H}$ bonds in the sample, which are abundant in fatty acids. Hence, lipid bodies emitted significantly stronger anti-Stokes signal than other cell compartments (Fig. 6E). A spatial overlay (Fig. 6D-F) of the recorded fluorescence and CARS images revealed a good co-localization of the observed bright dotted structures in both imaging modalities, further evidence that the NR positive structures were lipid bodies. The weak background signal in the CARS images could be partly assigned to dark and readout noise of the camera as well as to off-resonantly driven vibrational transitions in the sample. Since data gained by fluorescence and CARS microscopy suggested that NR was localized within lipid bodies highly enriched in triglycerides, transmission electron microscopy (TEM) was used to assess whether under the applied culture conditions, HEI-OC1 cells featured lipid bodies. When electron micrographs of HEI-OC1 cells were examined, osmium tetroxide contrasted lipid bodies, were frequently seen throughout the cytoplasm (Fig. 6I, arrowheads). Taken together, only diffusion of NR could explain the observed staining pattern, in the conditions of endocytosis inhibition.

Performing the same study at $37^{\circ} \mathrm{C}$, the pattern differed only in the magnitude of the NR staining, which was found to be stronger when performed at $37^{\circ} \mathrm{C}$. This might be related to a faster diffusion of the dye due to temperature increase.

In order to assess the localization patterns of $\mathrm{DiO}$ and NR when endocytosis is running on full scale, cells were incubated with NR/ DiO-loaded LNCs for $8 \mathrm{~h}$ on $37{ }^{\circ} \mathrm{C}$. NR revealed similar staining patterns as previously, while $\mathrm{DiO}$ fluorescence from distinct spots throughout the cytoplasm was observed (Fig. 6G). Strikingly the localizations of $\mathrm{DiO}$ and NR were different and not colocalized (Fig. 6G-H, insert in $\mathrm{H}$ and corresponding intensity profile), meaning that the dyes were located in different structures. Since DiO was only observed in experimental conditions allowing endocytosis and was shown to stay confined within the LNCs, it is likely that DiO fluorescence originates from endolysosomal compartments containing LNCs.

\section{Discussion}

Investigating the in vitro transfer of a drug or a dye is not an easy task because the separation between the nanocarrier suspension and the external medium has to be well controlled. When the external medium is water or buffer, separation between the nanocarrier suspension and the external medium was often successfully achieved using dialysis devices [20,21]. Nevertheless, dialysis exhibits two major drawbacks: this method is often used for purification (therefore passive release or transfer could be initiated during purification) [22] and poor in vitro/in vivo correlation is observed due to the presence of a physical barrier. In vivo, there is no artificial border between the nanocarriers and the biological fluids. The present study reports an 

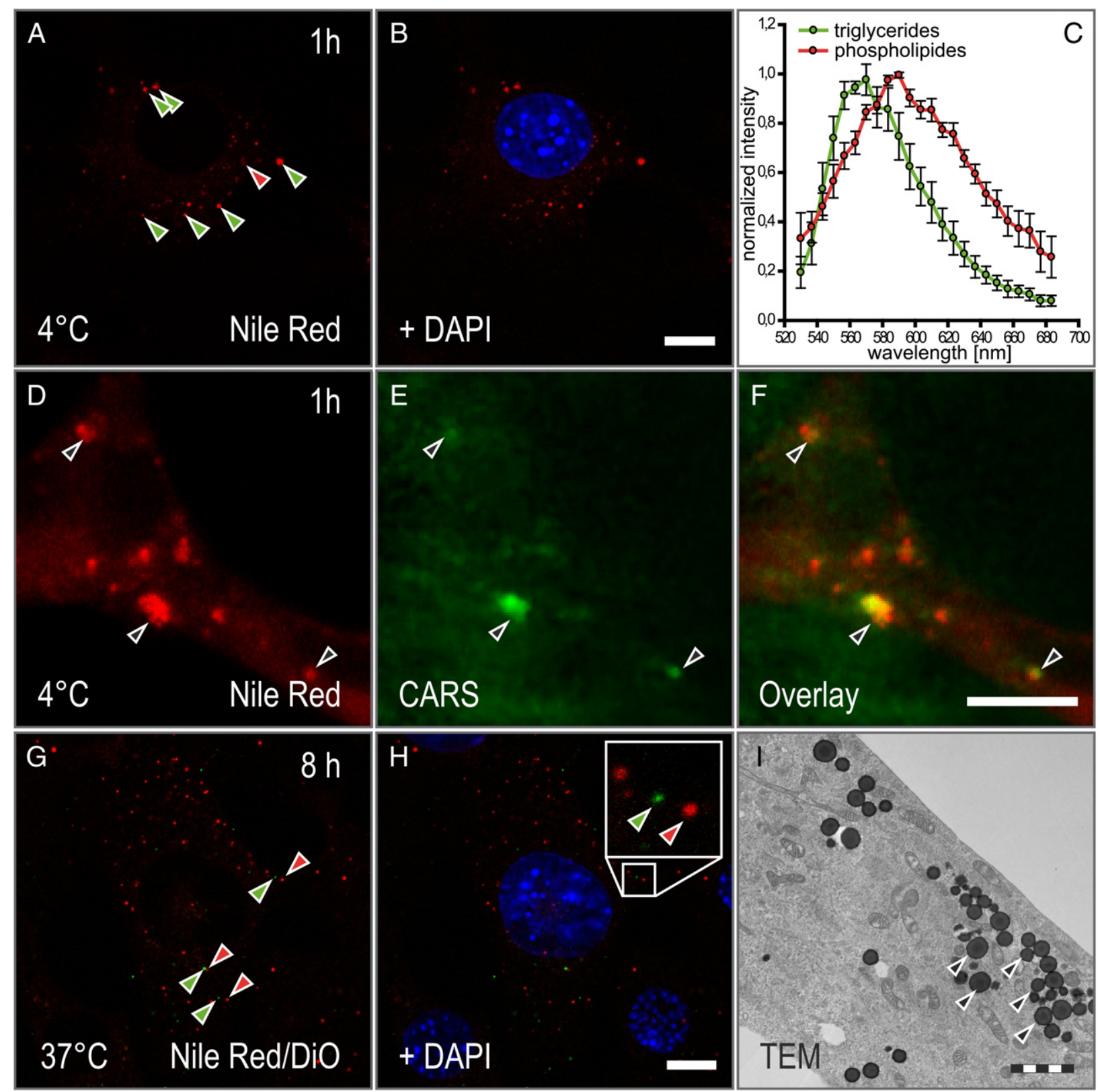

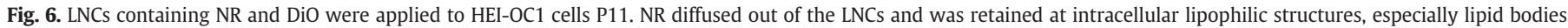

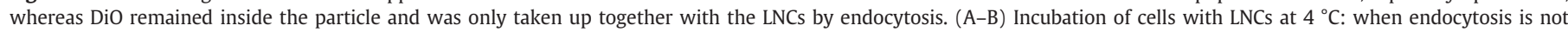

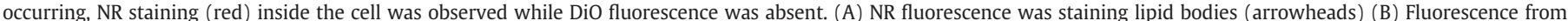

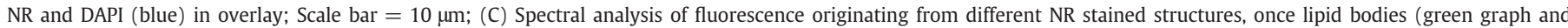

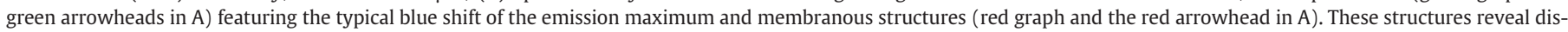

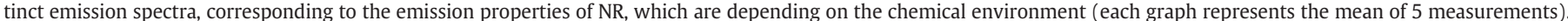

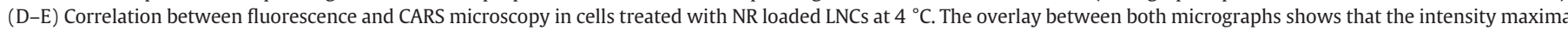

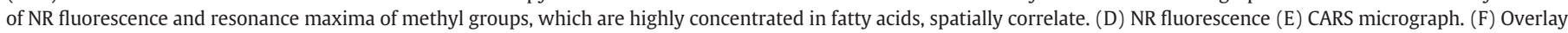

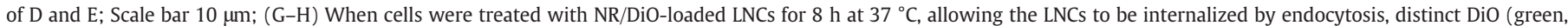

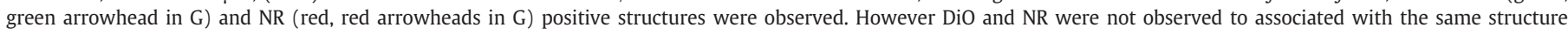

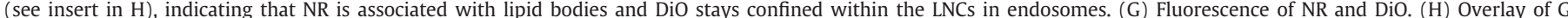

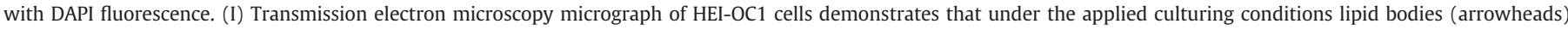
are found frequently in the cytoplasm of HEI-OC1 cells. Scale bar $=2 \mu \mathrm{m}$.

innovative protocol to assess the transfer of lipophilic fluorescent dye from a nanocarrier to a LC, without any physical separation. To recover the two non-miscible media, centrifugation was performed and fluorescence was used to determine the dye proportion in each compartment, confirming the labeling stability of the fluorescent nanocarriers.

Since no barrier separated the 2 media, fluorescence in LC might be due to the presence of LNCs in the oil phase caused by a non-optimal mechanical separation using centrifugation. It was therefore necessary to determine the LNC concentration in the aqueous phase before and after centrifugation. If a perfect separation was obtained, fluorescence in LC could be only due to the presence of lipophilic fluorescent dyes transferred from LNCs to the external oil compartment. The nanocarrier concentration (in particle/volume unit) is difficult to determine exactly. Some techniques can be used such as static light scattering with the determination of nano-object molecular weights [23] or turbidimetry to determine nanoparticle concentration (in weight/volume unit) 
[22]. DCR was used in this study as a new method for LNC concentration evaluation. To our knowledge, it was the first time this light scattering parameter was used for this purpose. Based on DCR versus LNC concentrations as titration curves (Appendix 1, Fig. S1), a good separation was carried out between LNC suspension and oil. Indeed, similar DCR value (for a given LNC size and PdI) was observed before and after centrifugation, showing no change in the LNC concentration in suspension.

A correlation was observed between the dye localization inside the LNCs and the transfer from LNCs to LC. The LNC structure is basically an oil core, surrounded by an organized structure of lecithin molecules and PEGylated surfactants suspended in water. NR and 6 -Cou should be localized inside the core of LNCs (according to tensiometry measurements, Fig. 4), without any interaction with the shell surfactants, allowing the diffusion of these two dyes from LNCs to other external lipophilic compartments as previously described.

The transfer was observed in a partition coefficient dependent manner, with separation between the two oil phases: LNCs and LC. The amount of dye transferred depended on the oil volumes while no dependency was observed as a function of the dye concentration and the LNC size. The transferred amounts were also depended on the affinity of the dye with the acceptor compartment: NR was not transferred to PFCE, due to its non-solubility, but can be transferred in a large variety of oil such as Labrafac, Captex200, Captex300 and EO (Supplementary information, Fig. S3). Finally, since the distance between LNCs and LC increased with dilution, NR transfer decreased whatever the initial localization of NR (LNCs or LC) (Fig. S2). Surprisingly, when the transfer experiments were performed with the ratio $\mathrm{R}=1 / 1$ ( similar amounts of Labrafac in LNC and in LC), the NR separation was not equivalent in LNC and LC, i.e. $50 \%$ of NR in each compartment. The hydrophobic core of LNC was not only composed of Labrafac but there were the hydroxystearate part of Kol and the lipophilic part of Lip. So the lipophilic volume of the LNC compartment was higher than LC. In addition, as shown using various oils, the NR proportion in each compartment after the transfer was modulated owing to the NR solubility in the specific oil. The higher quantity of NR in LNC than in LC after transfer with a ratio $R=1 / 1$ could be explained by a higher hydrophobic volume in LNC compartment and a higher NR solubility in LNC, due to the impact of the lipophilic part of surfactants. The transfer of NR or 6-Cou has already been observed by others when the dyes were loaded in different nanoparticles. Liposome dispersion in a PLGA nanoparticle suspension revealed a NR transfer from polymeric nanoparticles to liposomes which were acting as acceptor compartment, while no release was observed to aqueous solution (without liposome) [24]. When NR was loaded in liposomes, Petersen et al. showed the transfer of the lipophilic marker to large oil emulsions using flow cytometry [25].

No transfer was observed from loaded LNCs to LC with DiI, DiD and DiO whereas transfer occurred with NR and 6-Cou. Solubilities in water are below 1 and $1.7 \mu \mathrm{g} \cdot \mathrm{mL}^{-1}$ for NR and 6-Cou, respectively $[26,27]$ and Dil or another carbocyanine family dye, $1,1^{\prime}$-dioctadecyl$3,3,3^{\prime}, 3^{\prime}$-tetramethylindotricarbocyanine iodide (DiR) is traditionally used at about a $2 \mu \mathrm{g} \cdot \mathrm{mL}^{-1}$ concentration in aqueous buffer [28]. Difference in transfer behavior cannot be explained by difference in water solubilities of dyes. A higher solubility would have facilitated the transfer process from LNC to LC, and reversely. Due to their amphiphilic structures and properties (Fig. 4 and Appendix 2), DiI, DiD and certainly DiO, should participate in the formulation process (phase-inversion process) and were likely located and trapped in the surfactant shell around the oil core. Thus, the confinement of indocarbocyanine dyes in the surfactant shell can explain the absence of transfer from the LNCs (Appendix 2) and the absence of uptake of Dil and DiI in the LNCs, when present in the LC. In this case, LNC breaking could cause dye liberation, and reciprocally released dye from LNCs would indicate the LNC breakdown. Similar results were observed when Dil was loaded in liposomes, where there was no transfer from liposome to large oil emulsions [25]. Since no physical barrier separated the LNC suspension and the LC, NR and 6-Cou transfer could also be due to the direct contact of the LNCs and LC as assumed in literature $[25,29,30]$. Research is ongoing about this major issue. As the use of dyes in the pharmaceutical field is becoming increasingly common, it is important to note that a dye should not be chosen purely for its fluorescence properties such as wavelengths, quantum yield, etc. As shown in this study, a dye which is not covalently bound to nanocarrier should also be chosen for its physicochemical properties and location inside the nanocarrier.

Texier et al. recently reported the loading of DID and DiR inside lipid (mixture of soybean oil and Suppocire ${ }^{\circledR} \mathrm{NC}$ ) nanoparticles [31]. These nanocarriers were stabilized by a lecithin and Myrj® 53 surfactant layer. Depending on the encapsulation rate used, between 3 and 53 dye molecules were located in nanoparticles, and they concluded that the dyes were located in the core of nano-objects due to a constant zeta potential $(\mathrm{Pz})$ regardless of the dye concentration used. The same result was observed with Labrafac-based LNCs with DiI, DiD and DiO encapsulation. No difference was observed concerning Pz value $(-5 \mathrm{mV})$ compared to non-loaded LNCs (whatever the dye nature and the LNC size). Evaluation of the dye molecule numbers per LNC were assessed with the same assumption used by Texier et al. [31] (see appendix 3 for the calculation). There were about 1, 21 and 127 dye molecules per LNC, for 27, 65 and 110-nm Z-Ave LNC, respectively, in a similar range reported in literature [31]. The number of dye molecule was also compared to the number of surfactant molecules per LNC. The dye/surfactant ratio per LNC corresponded to about $0.05,0.11$ and $0.21 \%$ for 27,65 and 110-nm Z-Ave LNC, respectively, regardless of the dye nature (there were about 2200 , 19,300 and 59,900 surfactant molecules at the interface of one LNC). The number of dye molecules is too low, compared to the number of surfactant molecules, in one LNC to influence the Pz value. In addition, PEG chains (660 Da) of Kol surfactant, located in water, surrounded LNCs. This PEG shell could also have influence on the overall zeta potential hiding the charges located at the interface as shown by Vonarbourg et al. [32].

Finally, dye transfer was also demonstrated in cell culture. DiO-loaded LNCs were internalized after an $8 \mathrm{~h}$-incubation at $37^{\circ} \mathrm{C}$, while the presence of NR was observed inside cells after 1 h-incubation at $4{ }^{\circ} \mathrm{C}$. The diffusion of NR from the LNCs to HEI-OC1 cells, where NR accumulates, was also increased with temperature, suggesting a diffusion process that is temperature dependent. As mentioned before, NR transfer could depend on the contact between LNCs and cells. Nevertheless, the location of NR in lipid bodies inside the cells demonstrated that direct contact is not necessary since a cell membrane (strongly coated with glycoproteins) separates lipid bodies from the LNCs with two aqueous phases on either side. This permeation of the dyes across cell membrane is still not understood and will be addressed in future studies. The cell study was performed with one cell line (HEI-OC1) and needs to be repeated with others to confirm the general feature of the fluorescence labeling using LNC. It should also confirm the cellular trafficking results obtained with mesenchymal stromal cells [33], Caco-2 cells [6,34,35], macrophages [5], malignant rat-glioma cells (F98) [9], etc. Our observation is consistent with previous literature. Using solid lipid nanoparticles or nanostructured lipid carriers for in vitro cell culture and in vivo studies, the transfer of lipophilic markers was observed without the endocytosis of the nanocarriers. 6-Cou was transferred to A30 cells (lung cell line) in a similar manner when loaded in solid lipid nanoparticles, after $45-\mathrm{min}$ incubation at $32{ }^{\circ} \mathrm{C}$. In addition, performed at $4{ }^{\circ} \mathrm{C}$ when endocytosis is inhibited, the presence of 6-Cou was also observed in A30 cells [29]. Using confocal laser scanning microscopy, Tian et al. demonstrated the transfer of 6-Cou from nanostructured lipid carriers to corneal epithelia after the in vivo treatment of rabbit's eyes by the accumulation of the lipophilic marker in the lipophilic cells compartments (e.g. lipid membranes, subcellular vesicles) [30]. All these observations underline that for 
lipophilic dyes such as NR and Cou-6, endocytosis of the nano-sized carrier is not required for cellular uptake.

A new tool to show the stability of the fluorescence labeling of nanocarriers is proposed here. Based on these results, the labeling of LNCs using the indocarbocyanine dye family (Dil, DiO or DiD) will provide a more reliable means for fluorescence imaging of these and other lipophilic nanocarriers, compared to labeling using NR and 6-Cou.

\section{Conclusion}

It was demonstrated that dyes like NR and 6-Cou could be transferred from the LNCs to an oily medium or can enter blank LNCs from oily compartment. This behavior follows a partition property, being independent on changes of the dye concentration but dependent on oil volume modifications. On the other hand, no transfer of indocarbocyanine dyes occurred. These two antagonistic properties can be explained by different dye localization inside the nanocarrier. NR and 6-Cou localized in the core of the LNCs could be transferred in the presence of an external lipophilic compartment. Whereas DiI, DiO and DiD, trapped in the shell of the LNCs, could not be transferred, even in the presence of an external lipophilic compartment. These two behaviors were confirmed by cell culture experiments. The presence of NR inside lipophilic bodies of HEI-OC1 cells was observed, without LNC uptake. DiO-loaded LNCs have to be internalized to carry DiO to intracellular compartments, a further evidence of absence of transfer. The irreversible labeling using DiI, DiO or DiD will confirm the nanocarrier location in the cell (assuming the nanocarrier integrity) using fluorescence imaging techniques. In summary, a dye has to be chosen for its physico-chemical properties and location inside the nanocarrier, not only for its fluorescence properties, but also in order to guarantee unbiased experimental data.

This new protocol could be used for other nanocarriers and hydrophobic compartments as long as these two media can be separated by centrifugation without any medium interaction or nanocarrier degradation. In addition, drug-loaded nanocarriers could be tested to determine the drug release to hydrophobic media, and to confirm the influence of drug solubilization or trapping inside the nanocarrier. This new approach could have an important impact for in vivo experiments, which would shed more light on the mechanisms of drug release to fat or to epithelial cells after administration.

\section{Acknowledgments}

Authors wish to acknowledge the European Community (NANOEAR program: NMP-2004-34151, LYMPHOTARG program: EuroNanoMed ERA-NET 09), Austrian Science Fund (FWF CARS Project, FWF Project Nr. P22085) and Med El, Innsbruck, Austria for providing financial support for this work. Authors also thank Dr. Frederico Kalinec (Los Angeles, USA) for providing the HEI-OC1 cells.

\section{Appendix A. Supplementary data}

Supplementary data to this article can be found online at http:// dx.doi.org/10.1016/j.jconrel.2013.06.014.

\section{References}

[1] S. Gross, D. Piwnica-Worms, Molecular imaging strategies for drug discovery and development, Curr. Opin. Chem. Biol. 10 (2006) 334-346.

[2] K. Licha, C. Olbrich, Optimal imaging in drug discovery and diagnostic applications, Adv. Drug Deliv. Rev. 57 (2005) 1087-1108.

[3] J. Willmann, N. van Bruggen, L.M. Dinkelborg, S.S. Gambhir, Molecular imaging in drug development, Nat. Rev. Drug Discov. 7 (2008) 591-607.

[4] P.P. Ghoroghchian, M.J. Therien, D.A. Hammer, In vivo fluorescence imaging: a personal perspectives, Wiley Interdiscip. Rev. Nanomed. Nanobiotechnol. 1 (2) (2009) 156-167.
[5] E. Almouazen, S. Bourgeois, A. Boussaid, P. Valot, C. Malleval, H. Fessi, S. Nataf, S. Briancon, Development of a nanoparticle-based system for the delivery of retinoic acid into macrophages, Int. J. Pharm. 430 (1-2) (2012) 207-215.

[6] M. Gaumet, R. Gurny, F. Delie, Localization and quantification of biodegradable particles in an intestinal cell model: the influence of particle size, Eur. J. Pharm. Sci. 36 (4-5) (2009) 465-473.

[7] A.N. Jatariu Cadinoiu, M.N. Holban, C.A. Peptu, A. Sava, M. Costuleanu, M. Popa, Double crosslinked interpenetrated network in nanoparticle form for drug targetingpreparation, characterization and biodistribution studies, Int. J. Pharm. 436 (1-2) (2012) 66-74.

[8] D. Ding, J. Wang, Z.S. Zhu, R.T. Li, W. Wu, B.R. Liu, X.Q. Jiang, Tumor accumulation, penetration, and antitumor response of cisplatin-loaded gelatin/poly(acrylic acid) nanoparticles, ACS Appl. Mater. Interfaces 4 (3) (2012) 1838-1846.

[9] A. Paillard, F. Hindré, C. Vignes-Colombeix, J.-P. Benoit, E. Garcion, The importance of endo-lysosomal escape with lipid nanocapsules for drug subcellular bioavailability, Biomaterials 31 (2010) 7542-7554.

[10] B. Heurtault, P. Saulnier, B. Pech, J.-E. Proust, J. Richard, J.-P. Benoit, Patent No. WO02688000. (2000).

[11] B. Heurtault, P. Saulnier, B. Pech, J.-E. Proust, J.-P. Benoit, A novel phase inversionbased process for the preparation of lipid nanocarriers, Pharm. Res. 19 (6) (2002) 875-880.

[12] N.T. Huynh, C. Passirani, P. Saulnier, J.-P. Benoit, Lipid nanocapsules: a new platform for nanomedecine, Int. J. Pharm. 379 (2) (2009) 201-209.

[13] G.M. Kalinec, P. Webster, D.J. Lim, F. Kalinec, A cochlear cell line as an in vitro system for drug ototoxicity screening, Audiol. Neurootol. 8 (4) (2003) 177-189.

[14] M. Müller, A. Zumbusch, Coherent anti-stokes Raman scattering microscopy, Chem. Phys. Chem. 8 (15) (2007) 2156-2170.

[15] A. Jesacher, C. Roider, S. Khan, G. Thalhammer, S. Bernet, M. Ritsch-Marte, Contrast enhancement in widefield CARS microscopy by tailored phase matching using a spatial light modulator, Opt. Lett. 36 (2011) 2245-2247.

[16] C.O. Pritz, M. Bitsche, W. Salvenmoser, J. Dudas, A. Schrott-Fischer, R. Glueckert, Endocytic trafficking of silica nanoparticles in a cell line derived from the organ of Corti, Nanomedicine (Lond.) 0 (2012) 1-14

[17] K. Shinoda, H. Saito, The stability of O/W type emulsions as functions of temperature and the HLB of emulsifiers: the emulsification by PIT-method, J. Colloid Interface Sci. 30 (1969) 258-263.

[18] I. Foissner, Fluorescent phosphocholine-a specific marker for the endoplasmic reticulum and for lipid droplets in Chara internodal cells, Protoplasma 238 (2009) 47-58

[19] P. Greenspan, S.D. Fowler, Spectrofluorometric studies of the lipid probe, nile red J. Lipid Res. 26 (1985) 781-789.

[20] S. Vrignaud, N. Anton, P. Gayet, J.-P. Benoit, P. Saulnier, Reverse micelle-loaded lipid nanocarriers: a novel drug delivery system for the sustained release of doxorubicin hydrochloride, Eur. J. Pharm. Biopharm. 79 (2011) 197-204.

[21] A. Lamprecht, Y. Bouligand, J.-P. Benoit, New lipid nanocapsules exhibit sustained release properties for amiodarone, J. Control. Release 84 (2002) 59-68.

[22] H. Meyer, T. Stöver, F. Fouchet, G. Bastiat, P. Saulnier, W. Baümer, T. Lenarz, V. Scheper, Lipidic nanocapsule drug delivery: neuronal protection for cochlear implant optimization, Int. J. Nanomedicine 7 (2012) 2449-2464.

[23] G. Bastiat, B. Grassl, A. Khoukh, J. Francois, Study of sodium dodecyl sulfatepoly(propylene oxide) methacrylate mixed micelles, Langmuir 20 (2004) 5759-5769.

[24] P. Pietzonka, B. Rothen-Rutishauser, P. Langguth, H. Wunderli-Allenspach, E. Walter, H.P. Merkle, Transfer of lipophilic markers from PLGA and polystyrene nanoparticles to Caco-2 monolayers mimics particle uptake, Pharm. Res. 19 (5) (2002) 595-601

[25] S. Petersen, A. Fahr, H. Bunjes, Flow cytometry as a new approach to investigate drug transfer between lipid particles, Mol. Pharm. 7 (2) (2010) 350-363.

[26] A. Hawe, M. Sutter, W. Jiskoot, Extrinsic fluorescent dyes as tools for protein characterization, Pharm. Res. 25 (7) (2008) 1487-1499.

[27] P.M. Boisde, W.C. Meuly, Coumarin, Kirk-Othmer Encyclopedia of chemica technology, 7 (4th ed.), John Wiley \& Sons, 1993, pp. 647-658.

[28] F. Lassailly, E. Griessinger, D. Bonnet, "Microenvironmetal contaminations" unduced by fluorescent lipophiic dyes used for noninvasive in vitro and in vivo cell tracking, Blood 115 (26) (2010) 5347-5354.

[29] I. Rivolta, A. Panariti, B. Lettiero, S. Sesana, P. Gasco, M.R. Gasco, M. Masserini, G Miserocchi, Cellular uptake of coumarin-6 as a model drug loaded in solid lipid nanoparticles, J. Physiol. Pharmacol. 62 (1) (2011) 45-53.

[30] B.-C. Tian, W.J. Zhang, H.-M. Xu, M.-X. Hao, Y.-B. Liu, X.-G. Yang, W.-S. Pan, X.-H. Liu, Further investigation of nanostructured lipid carriers as an ocular delivery system: in vivo transcorneal mechanism and in vitro release study, Colloids Surf. B 102 (2013) 251-256.

[31] I. Texier, M. Goutayer, A. Da Silva, L. Guyon, N. Djaker, V. Josserand, E. Neumann, J Bibette, F. Vinet, Cyanine-loaded lipid nanoparticles for improved in vivo fluorescence imaging, J. Biomed. Opt. 14 (5) (2009) 054005.

[32] A. Vonarbourg, P. Saulnier, C. Passirani, J.-P. Benoit, Electrokinetic properties of noncharged lipid nanocapsules: influence of the dipolar distribution at the interface, Electrophoresis 26 (11) (2005) 2066-2075.

[33] M. Roger, A. Chevreul, M.-C. Venier-Julienne, C. Passirani, L. Sindji, P. Schiller, C. Montero-Menei, P. Menei, Mesenchymal stem cells as cellular vehicles for delivery of nanoparticles to brain tumors, Biomaterials 31 (2010) 8393-8401.

[34] E. Roger, F. Lagarce, E. Garcion, J.-P. Benoit, Reciprocal competition between lipid nanocapsules and P-gp for paclitaxel transport across Caco-2 cells, Eur. J. Pharm. Sci. 40 (2010) 422-429.

[35] E. Roger, F. Lagarce, E. Garcion, J.-P. Benoit, Lipid nanocarriers improve paclitaxel transport throughout human intestinal epithelial cells by using vesicle-mediated transcytosis, J. Control. Release 140 (2009) 174-181. 